\title{
D04 Joint Inversion of Production Data and 4D-seismic Attributes - Parameterization Issues
}

M. Le Ravalec, V. Gervais \& F. Roggero

IFP, $1 \& 4$ avenue de Bois Préau 9852 Rueil-Malmaison, France

The purpose of this work is to characterize reservoir models from the available production data and 4D-seismic attributes. Production data and 4D-seismic attributes are very complementary. Production data are sparsely distributed in space while highly distributed in time. 4D-seismic data are just the opposite: highly distributed in space while sparsely distributed in space. In addition, the number of data is much greater for seismic than for production. Thus, questions arise about the way to integrate these data into reservoir models. Shall we use the same kind of parameterization techniques?

As production and 4D-sesimic data are non linearly related to petrophysical properties, their integration is formulated as an inverse problem solved using optimization techniques. Conditional models are derived from the minimization of the misfit between numerically predicted flow responses and field measurements. This process consists of sequentially varying the initial petrophysical model until a reasonable match is achieved. A great deal of work has been dedicated to this subject over the last three decades. It motivated the development of geostatistics-based parameterization techniques, which allow for modifying a petrophysical model from a limited number of parameters while preserving its two-order statistics : the pilot point method, the gradual deformation method and the probability perturbation method. All of these geostatistics-based optimization processes suffer from slow convergence rates. The first iterations are often efficient, but then improving the matching gets more demanding. This difficulty is even more patent when jointly handling production and 4D-seismic data.

Roggero et al. (2007) studied the large, complex and faulted turbidite Girassol field located offshore Angola. They evidenced the need for locally perturbing the petrophysical properties to better match 4D-seismic data. In this context, a first prospect to improve the matching methodology consists of judiciously defining the sub-domains to be changed. The identification of the streamlines arriving to the areas exhibiting a significant 4Dseismic mismatch yields upstream influence zones, which also contribute to the observed mismatch. They provide the sub-domains of interest. The second prospect focuses on the parameterization itself. We then develop a new parameterization technique to change the mean of petrophysical properties over the selected sub-domains. An example is presented to demonstrate the applicability of the proposed matching methodology.

Roggero, F., Ding, D.Y., Berthet, P., Lerat, O., Cap, J., and Schreiber, P.E.: Matching of production history and 4D seismic data - Application to the Girassol field, offshore Angola. SPE ATCE, Anaheim, CA, USA, SPE 109929 (2007) 